\title{
DISTRIBUTIONAL AND ENTIRE SOLUTIONS OF LINEAR FUNCTIONAL DIFFERENTIAL EQUATIONS
}

\author{
JOSEPH WIENER \\ Department of Mathematics \\ Pan American University \\ Edinburg, Texas 78539 \\ (Received February 26, 1982)
}

\begin{abstract}
A unified approach to the study of generalized-function and entire solutions to linear functional differential equations with polynomial coefficients is suggested.
\end{abstract}

KEY WORDS AND PHRASES. Functional Differential Equations, Distributional Solutions, Entire Solutions.

1980 Mathematics Subject Classification Codes. 34K25, 34K99, 46F10, 30D05.

1. INTRODUCTION AND PRELIMINARIES.

An interesting survey of recent results on entire solutions of ordinary differential equations with polynomial coefficients is given in [1]. In the present paper we continue the study of distributional solutions to linear functional differential equations (FDE) in accordance with the concepts outlined in [2] and [3]. There are profound and close links between spaces of generalized and entire functions [4]. Therefore, the basic ideas in the method of proof may also be applied to the study of entire solutions to linear FDE, especially with linear transformations of the argument. We investigate such linear homogeneous FDE with infinitely smooth coefficients that have solutions in the class of singular functionals which is impossible for analogous ordinary differential equations. Solutions of linear FDE with countable sets of variable argument deviations are considered in the generalizedfunction space $\left(s_{0}^{\beta}\right)^{\prime}$ conjugate to the space $s_{0}^{\beta}$ of testing functions $\phi(t)$ that satisfy the restriction [4]

$$
\left|\phi^{(n)}(t)\right| \leq a c^{n} n^{n \beta}, \beta>1 .
$$


In the sequel, $\delta^{(n)}$ denotes the nth derivative of the Dirac $\delta$ measure and $\langle f, \phi\rangle$ is the value of the functional $f$ applied to the testing function $\phi$ of the real variable $t$. The norm of a matrix is defined to be

$$
\|A\|=\max _{i} \sum_{j}\left|a_{i j}\right|
$$

and $E$ is the identity matrix. In [2], it has been proved that under certain conditions the system

$$
\sum_{i=0}^{\infty} \sum_{j=0}^{m}\left(A_{i j}+t B_{i j}\right) x^{(j)}\left(\lambda_{j} t\right)=t x(\lambda t)
$$

has a solution

$$
x(t)=\sum_{n=0}^{\infty} x_{n} \delta^{(n)}(t)
$$

in $\left(s_{0}^{\beta}\right)^{\prime}$ with arbitrary $\beta>1$. To ensure the convergence of series $(1.1)$, it is sufficient to require that for $n \rightarrow \infty$ the vectors $x_{n}$ satisfy the inequalities

$$
\left\|x_{n}\right\| \leq b d^{n} n^{-n \rho}, \rho>1
$$

since

$$
\begin{aligned}
& \| \sum_{n=0}^{\infty}\left\langle x_{n} \delta^{(n)}(t), \phi(t)>\|=\| \sum_{n=0}^{\infty}(-1)^{n} \phi^{(n)}(0) x_{n} \| \leq\right. \\
& \leq \sum_{n=0}^{\infty}\left|\phi^{(n)}(0)\right|\left\|x_{n}\right\| \leq a b \sum_{n=0}^{\infty}\left(c d n^{\beta-\rho}\right)^{n}<\infty,
\end{aligned}
$$

for $\beta<\rho$. If series (1.1) converges, its sum represents the general form of a linear functional in $\left(s_{0}^{\beta}\right)^{\prime}$ with the support $t=0$ [5]. Some recent developments in astrophysics posed new problems about the existence of distributional solutions also to certain integral equations [2].

2. EXISTENCE OF DISTRIBUTIONAL SOLUTIONS.

We look for solutions of the form (1.1) to the system

$$
\sum_{i=0}^{\infty} \sum_{j=0}^{m} A_{i j}(t) x^{(j)}\left(\lambda_{i j}(t)\right)=0 .
$$

LEMMA. If $t_{0}$ is a fixed point of the function $\lambda(t) \varepsilon c^{1}(-\infty, \infty)$ and $\lambda^{\prime}\left(t_{0}\right) \neq 0$, then in some neighborhood of $t_{0}$,

$$
\delta^{(n)}\left(\lambda(t)-t_{0}\right)=\delta^{(n)}\left(t-t_{0}\right) /\left(\lambda^{\prime}\left(t_{0}\right)\right)^{n}\left|\lambda^{\prime}\left(t_{0}\right)\right|
$$


PROOF. Since $\lambda\left(t_{0}\right)-t_{0}=0$,

$$
\lambda(t)-t_{0}=\left(t-t_{0}\right) \psi(t), \psi\left(t_{0}\right)=\lambda^{\prime}\left(t_{0}\right) .
$$

There exists a neighborhood $D_{0}$ of $t_{0}$ at all points of which $\psi(t) \neq 0, t \neq t_{0}$, for assuming the opposite we find a sequence $t_{0 v} \rightarrow t_{0}$ such that $\lambda\left(t_{0 \nu}\right)-\lambda\left(t_{0}\right)=0$ and, hence, $\lambda^{\prime}\left(t_{0}\right)=0$.

In $D_{0}$ for $t \neq t_{0}$,

$$
\delta^{(n)}\left(\lambda(t)-t_{0}\right)=\delta^{(n)}\left(\psi(t)\left(t-t_{0}\right)\right)=0 .
$$

But for $t=t_{0}$,

$$
\delta^{(n)}\left(\lambda(t)-t_{0}\right)=\delta^{(n)}\left(\lambda^{\prime}\left(t_{0}\right)\left(t-t_{0}\right)\right) .
$$

Thus, (2.3) holds for all $t \varepsilon D_{0}$ and it remains to observe that $\delta^{(n)}(c t)=\delta^{(n)}(t) / c^{n}|c|$.

THEOREM 2.1. Let $(2.1)$, in which $x(t)$ is an $r$-vector and $A_{i j}(t)$ are $r \times r-$ matrices, satisfy the following hypotheses.

(i) The real-valued functions $\lambda_{1 j}(t) \varepsilon c^{1}$ have a common fixed point $t_{0}$ and $0<\left|\lambda_{00}^{\prime}\left(t_{0}\right)\right|<1,\left|\lambda_{i j}^{\prime}\left(t_{0}\right)\right| \geq 1,1+j \geq 1$.

(ii) The coefficients $A_{i j}(t)$ are polynomials of degree not exceeding $p$ :

$$
A_{i j}(t)=\sum_{k=0}^{p} A_{i j k}\left(t-t_{0}\right)^{k}, A_{00}(t)=A\left(t-t_{0}\right)^{p}, p \geq 1 .
$$

(iii) The series $\sum_{i=1}^{\infty} \lambda_{i}^{-1} A_{i}$ converges where

$$
A_{i}=\underset{j, k}{\max }\left\|A_{i j k}\right\|, \lambda_{i}=\underset{j}{\inf } \mid \lambda_{i j}^{\prime}\left(t_{0}\right), i+j \geq 1 .
$$

(iv) The matrix $\mathrm{A}$ is nonsingular and

$$
c=\left|\lambda_{00}^{\prime}\left(t_{0}\right)\right|^{-p-1}\|A\|-\sum_{i>1}\left|\lambda^{\prime}{ }_{i 0}\left(t_{0}\right)\right|^{-p-1}\left\|A_{\text {iop }}\right\|>0 .
$$

Then, in some neighborhood of $t_{0}$, there exists a solution $x(t) \varepsilon\left(s_{0}^{\beta}\right)$ ' with arbitrary $\beta>1$ :

$$
x(t)=\sum_{n=0}^{\infty} x_{n} \delta^{(n)}\left(t-t_{0}\right) .
$$

PROOF. By virtue of (2.2) and the formula $t^{k} \delta^{(n)}(t)=(-1)^{k} n ! \delta^{(n-k)}(t) /(n-k) !$, for $n \geq k$, and 0 for $n<k$, we obtain the equations 


$$
\begin{aligned}
& \sum_{i, j, k}(-1)^{k} A_{i j k} \sum_{n+j \geq k}(n+j) ! x_{n} \delta^{(n+j-k)}\left(t-t_{0}\right) /(n+j-k) !\left|\alpha_{i j}\right| \alpha_{i j}^{n+j}=0, \\
& \alpha_{i j}=\lambda_{i j}^{\prime}\left(t_{0}\right)
\end{aligned}
$$

for the unknowns $x_{n}$ of the solution $x(t)$. Hence,

$$
\Sigma(-1)^{k}(n+k) !\left|\alpha_{i j}\right|^{-1} \alpha_{i j}^{-n-k} A_{i j k} x_{n+k-j}=0, n \geq 0
$$

which can be written as

$$
\begin{aligned}
& \sum_{k-j<p}(-1)^{p-k}(n+k) !\left|\alpha_{i j}\right|^{-1} \alpha_{i j}^{-n-k} A_{i j k} x_{n+k-j} /(n+p) !+ \\
& +\left(\sum_{i \geq 0}\left|\alpha_{i 0}\right|^{-1} \alpha_{i 0}^{-n-p} A_{i 0 p}\right) x_{n+p}=0 .
\end{aligned}
$$

Since $A_{00 k}=0(k<p)$, the first sum does not include terms with $\alpha_{00}$. According to (iv), the coefficients of $x_{n+p}$ are nonsingular matrices and

$$
\left\|\left(\sum_{i=0}^{\infty}\left|\alpha_{i 0}\right|^{-1} \alpha_{i 0}^{-n-p} A_{i 0 p}\right)^{-1}\right\|^{\prime} \leq c\|A\|\left|\alpha_{00}\right|^{n} .
$$

Consequent1y,

$$
\left\|x_{n+p}\right\| \leq \mu q^{n+p} \sum_{k=0}^{m+p-1}\left\|x_{n+k-m}\right\|, 0<q<1
$$

where $\mu$ is some positive constant. Using the notation

$$
M_{n}=\max _{0 \leq i \leq n}\left\|x_{i}\right\|
$$

we conclude from (2.4) that

$$
\left\|x_{n+p}\right\| \leq \mu(m+p) q^{n+p_{M+p-1}} .
$$

For large $n$, there is $\mu(m+p) q^{n+p} \leq 1$. Hence, $\left\|x_{n+p}\right\| \leq M_{n+p-1}$ and $M_{n+p}=M_{n+p-1}$. Thus, starting with some $N$,

$$
\mathrm{M}_{\mathrm{n}}=\mathrm{M}_{\mathrm{N}}, \mathrm{n} \geq \mathrm{N} \text {. }
$$

The application of $(2.6)$ to $(2.4)$ successively yields:

$$
\left\|x_{\mathrm{N}+\mathrm{p}+\mathrm{k}}\right\| \leq \mu(\mathrm{m}+\mathrm{p}) \mathrm{q}^{\mathrm{N}+\mathrm{p}_{\mathrm{M}_{\mathrm{N}}}} \text {, }
$$




$$
\begin{gathered}
\left\|x_{N+p+(m+p)+k}\right\| \leq \mu^{2}(m+p){ }_{q}{ }^{N+p} q^{N+p+(m+p)} M_{N} \\
\left\|x_{N+p+2(m+p)+k}\right\| \leq \mu^{3}(m+p){ }^{3} q^{N+q} q^{N+p+(m+p)} q^{N+p+2(m+p)} M_{N} \\
(0 \leq k \leq m+p-1) .
\end{gathered}
$$

The conjecture

$$
\left\|\mathrm{x}_{\mathrm{N}+\mathrm{p}+\mathrm{n}(\mathrm{m}+\mathrm{p})+\mathrm{k}}\right\| \leq \mu^{\mathrm{n}+1}(\mathrm{~m}+\mathrm{p})^{\mathrm{n}+1} \mathrm{q}^{\mathrm{n}(\mathrm{N}+\mathrm{p})+\mathrm{n}(\mathrm{n}+1)(\mathrm{m}+\mathrm{p}) / 2} \mathrm{M}_{\mathrm{N}}
$$

may readily be ascertained by induction for all $\mathrm{n}$ and the mentioned values of $\mathrm{k}$, and proves the theorem since the condition $0<q<1$ makes it more restrictive than $(1.2)$.

\section{EXISTENCE OF ENTIRE SOLUTIONS.}

We apply the method of the previous section to prove the existence of entire solutions of linear FDE with polynomial coefficients and to evaluate their order of growth.

THEOREM 3.1. Suppose the system

$$
x^{(P)}(t)=\sum_{i=0}^{\infty} \sum_{j=0}^{p} Q_{i j}(t) x^{(j)}\left(\lambda_{i j} t\right), x^{(j)}(0)=x_{j}, j=0, \ldots, p-1
$$

in which $Q_{i j}$ and $X$ are $(r \times r)$ - matrices, satisfies the following conditions:

(i) $Q_{i j}(t)$ are polynomials of degree not exceeding $m$;

(ii) $\lambda_{i j}$ are complex numbers such that

$$
0<q_{1} \leq\left|\lambda_{i j}\right| \leq 1,(j=0, \ldots, p-1), 0<q_{2} \leq\left|\lambda_{i p}\right| \leq q_{3}<1 ;
$$

(iii) the series $\Sigma Q^{(i)}$ converges, where $Q^{(i)}=\underset{j, k}{=\max _{\infty}}\left\|Q_{i j k}\right\|$ and $Q_{i j k}$ are the coefficients of $Q_{i j}(t)$, and $\sum_{i=0}^{\infty}\left\|Q_{i p}(0)\right\|<1$.

Then the problem has a unique holomorphich solution, which is an entire function or order not exceeding $m+p$.

PROOF. The expansions

$$
Q_{i j}(t)=\sum_{k=0}^{m} Q_{i j k} t^{k}, X(t)=\sum_{n=0}^{\infty} X_{n} t^{n}
$$

imply that

$$
x^{(p)}(t)=\sum_{n=0}^{\infty} X_{n+p} t^{n}(n+p) ! / n !
$$




$$
\begin{aligned}
& x^{(j)}\left(\lambda_{i j} t\right)=\sum_{n=0}^{\infty} \lambda_{i j}^{n} x_{n+j} t^{n}(n+j) ! / n ! \\
& Q_{i j}(t) x^{(j)}\left(\lambda_{i j} t\right)=\sum_{n=0}^{\infty} t^{n} \sum_{k=0}^{m} \lambda_{i j}^{n-k} Q_{i j k} X_{n+j-k}(n+j-k) ! /(n-k) !
\end{aligned}
$$

and yield the following recursion relations for the matrices $x_{n}$ :

$$
\begin{aligned}
(E- & \left.\sum_{i=0}^{\infty} \lambda_{i p}^{n} Q_{i p 0}\right) x_{n+p}=\sum_{i=0}^{\infty} \sum_{j=0}^{p-1} \sum_{k=0}^{m} \frac{n !(n+j-k) !}{(n+p) !(n-k) !} \lambda_{i j}^{n-k} Q_{i j k} X_{n+j-k} \\
& +\sum_{i=0}^{\infty} \sum_{k=1}^{m} \frac{n !(n+p-k) !}{(n+p) !(n-k) !} \lambda_{i p}^{n-k} Q_{i p k} X_{n+p-k}, n \geq 0
\end{aligned}
$$

Hypotheses (ii) and (iii) ensure the existence of the inverse matrices

$$
\begin{aligned}
\left(E-\sum_{i=0}^{\infty} \lambda_{i p}^{n} Q_{i p 0}\right)^{-1} \text { for al1 } n: & \\
& \left(E-\sum_{i=0}^{\infty} \lambda_{i p}^{n} Q_{i p 0}\right)^{-1}=\sum_{k=0}^{\infty}\left(\sum_{i=0}^{\infty} \lambda_{i p}^{n} Q_{i p 0}\right)^{k}, \\
& \left\|\left(E-\sum_{i=0}^{\infty} \lambda_{i p}^{n} Q_{i p 0}\right)^{-1}\right\| \leq\left(1-\sum_{i=0}^{\infty}\left\|Q_{i p 0}\right\|\right)^{-1} .
\end{aligned}
$$

Therefore, formulas (3.2) determine the coefficients $x_{n}$ uniquely and, since

$$
\begin{aligned}
& n !(n+j-k) ! /(n+p) !(n-k) ! \leq(n+p)^{-1}, 0 \leq j \leq p-1 \\
& n !(n+p-k) ! /(n+p) !(n-k) ! \leq 1,
\end{aligned}
$$

we obtain, by virtue of (iii),

$$
\left\|x_{n+p}\right\| \leq \frac{a}{n+p} \cdot \sum_{j=0}^{p-1} \sum_{k=0}^{m}\left\|x_{n+j-k}\right\|+b q_{3}^{n} \sum_{k=1}^{m}\left\|x_{n+p-k}\right\|
$$

For large $n$, there is $q_{3} \leq(n+p)^{-1}$ and

$$
\left\|x_{n+p}\right\| \leq c(n+p)^{-1} \sum_{k=0}^{m+p-1}\left\|x_{n+k-m}\right\| \text {. }
$$

Here $a, b, c$ are some positive constants. With the notation (2.5), it follows from (3.3) that

$$
\left\|x_{n+p}\right\| \leq c(m+p) M_{n+p-1} /(n+p) .
$$

Starting with some $N$,

$$
c(n+p) /(n+p) \leq 1,\left\|x_{n+p}\right\| \leq M_{n+p-1}, M_{n+p}=M_{n+p-1}
$$

and it remains to apply (2.6) successively to (3.3):

$$
\left\|x_{N+p+k}\right\| \leq c(m+p) M_{N} /(N+p),
$$




$$
\begin{gathered}
\left\|\mathrm{X}_{\mathrm{N}+\mathrm{p}+(\mathrm{m}+\mathrm{p})+\mathrm{k}}\right\| \leq \mathrm{c}^{2}(\mathrm{~m}+\mathrm{p})^{2} \mathrm{M}_{\mathrm{N}} /(\mathrm{N}+\mathrm{p})(\mathrm{N}+(\mathrm{m}+\mathrm{p})+\mathrm{p}), \\
\left\|\mathrm{X}_{\mathrm{N}+\mathrm{p}+2(\mathrm{~m}+\mathrm{p})+\mathrm{k}}\right\| \leq \mathrm{c}^{3}(\mathrm{~m}+\mathrm{p}){ }^{3} \mathrm{M}_{\mathrm{N}} /(\mathrm{N}+\mathrm{p})(\mathrm{N}+(\mathrm{m}+\mathrm{p})+\mathrm{p})(\mathrm{N}+2(\mathrm{~m}+\mathrm{p})+\mathrm{p}) \\
(0 \leq \mathrm{k} \leq \mathrm{m}+\mathrm{p}-1) .
\end{gathered}
$$

Now it can be proved easily that, for all $\mathrm{n}$,

Thus,

$$
\left\|x_{N+p+n(m+p)+k}\right\| \leq c^{n+1}(m+p)^{n+1} M_{N} / \prod_{i=0}^{n}(N+i(m+p)+p) .
$$

$$
\left\|x_{N+p+n(m+p)+k}\right\| \leq c^{n+1}(m+p) M_{N} / n !
$$

and the solution $X(t)$ is an entire function whose order of growth does not exceed $\mathrm{m}+\mathrm{p}$

THEOREM 3.2. If, in addition to the hypotheses of Theorem 3.1, the parameters $\lambda_{i j}(0 \leq j \leq p-1)$ are separated from unity: $0<q_{1} \leq\left|\lambda_{i j}\right| \leq q_{4}<1$, the solution of (3.1) is an entire function of zero order.

PROOF. Under the conditions of Theorem 2.1 , the system

$$
\sum_{i=0}^{\infty} \sum_{j=0}^{m} A_{i j}(t) X^{(j)}\left(\alpha_{i j} t\right)=0
$$

with real constants $\alpha_{i j}$,

$$
0<\left|\alpha_{00}\right|<1,\left|\alpha_{i j}\right| \geq 1,1+j \geq 1
$$

has a distributional solution (1.1), the coefficients $x_{n}$ of which satisfy inequalities (1.2) and are determined with the exactness to arbitrary $x_{0}, \ldots, x_{p-1}$ We apply to (3.4) the Laplace transformation assuming $\alpha_{i j}$ positive and retaining the same notation for $X(t)$ and its transform:

$$
x^{(p)}\left(s / \alpha_{00}\right)+\alpha_{00}^{p+1} A^{-1} \sum_{i+j>0} \sum_{k=0}^{p}(-1)^{p-k} \alpha_{i j}^{-j-1} A_{i j k}\left(s^{j} X\left(s / \alpha_{i j}\right)\right)(k)=0
$$

The substitutions $s / \alpha_{00}=t$, and $\alpha_{00} / \alpha_{1 j}=\lambda_{i j}$ reduce this equation to the form (3.1). This proves the theorem, since the transform of $\delta^{(n)}(t)$ is $s^{n}$ and the coefficients $x_{n}$ satisfy (2.7). These estimates use only the moduli of $\alpha_{1 j}$; hence, the parameters $\lambda_{i j}$ may be complex. Theorems 3.1 and 3.2 generalize the results of [6]. 
THEOREMS 3.3. The problem

$$
\begin{gathered}
F^{\prime}(z)=\sum_{i=0}^{\infty} A_{i}(z) F\left(z-a_{i}\right)+\sum_{i=0}^{\infty} B_{i}(z) F^{\prime}\left(z-b_{i}\right), \\
\lim _{\operatorname{Re} z \rightarrow-\infty} F(z)=F_{0}
\end{gathered}
$$

with $(r \times r)$ - matrices A, B, F has a unique holomorphic solution which is an entire function if:

(i) $A_{i}(z)=\sum_{k=1}^{m} A_{i k} e^{k z}, B_{i}(z)=\sum_{k=0}^{m} B_{i k} e^{k z}$;

$$
a_{i}, b_{i} \text { are complex numbers such that }
$$

$$
0 \leq \text { Rea }_{\mathbf{i}} \leq M_{1}<\infty, 0<M_{2} \leq \operatorname{Reb}_{i} \leq M_{3}<\infty ;
$$

(iii) the series $\Sigma A^{(i)}$ and $\sum B^{(i)} e^{-\operatorname{Reb}_{i}}$

converge where $A^{(i)}=\max _{k}\left\|A_{i k}\right\|, B^{(i)}=\max _{k}\left\|B_{i k}\right\|$, and $\sum_{i=0}^{\infty}\left\|B_{i}(0)\right\| e^{-R e b_{i}}<1$.

PROOF. The substitutions $t=e^{z}, \alpha_{i}=e^{-a_{i}}, \beta_{i}=e^{-b_{i}}$, and $F(z)=x(t)$ reduce (3.5) to (3.1) of the first order with the initial condition $X(0)=F_{0}$

\section{REFERENCES}

1. Fricke, G.H.; Roy, Ranjan and Shah, S. M. Bounded index entire solutions of ordinary differential equations and summability methods, Internat. J. Math. \& Math. Sci. 4 (3), (1981), 417-434.

2. Wiener, Joseph. Generalized-function solutions of linear systems, J. Differ. Equat. 38(2), (1980), 301-315.

3. Wiener, Joseph. Generalized-function solutions of differential and finctional differential equations, J. Math. Ana1. and Appl. 88(1), (1982), 170-182.

4. Gel'fand, I.M. and Shilov, G.E. Generalized functions, Vol. 2, Academic Press, New York, 1968.

5. Mityagin, B.S. On the infinitely differentiable function with the given values of the derivatives at a point, Dok1. Akad. Nauk SSSR 138(2), (1961), 289-292.

6. Wiener, Joseph. On the entire solutions of a linear functional differential system, Differ. Uravn. 13(3), (1977), 551-555. 


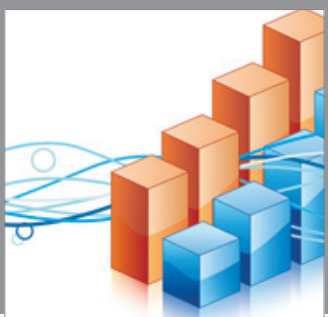

Advances in

Operations Research

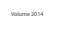

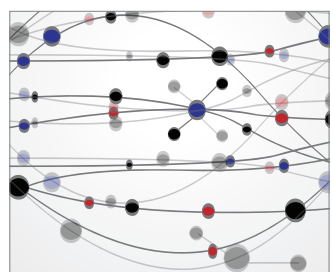

\section{The Scientific} World Journal
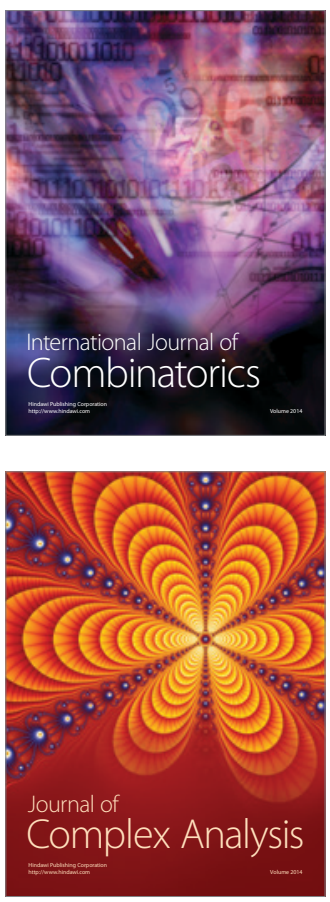

International Journal of

Mathematics and

Mathematical

Sciences
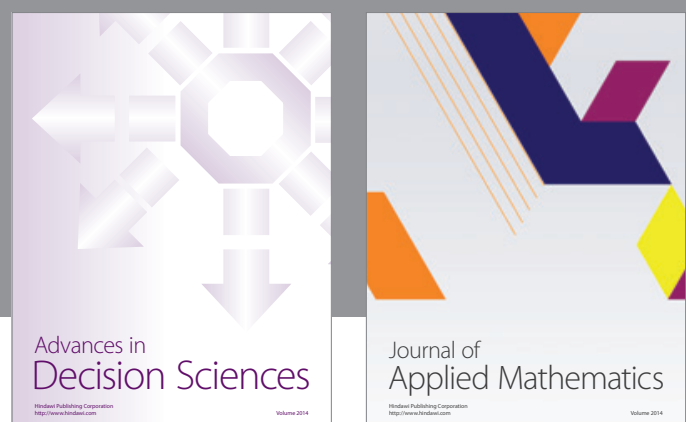

Journal of

Applied Mathematics
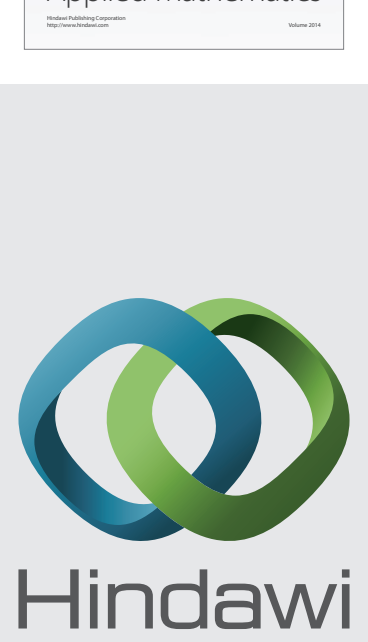

Submit your manuscripts at http://www.hindawi.com
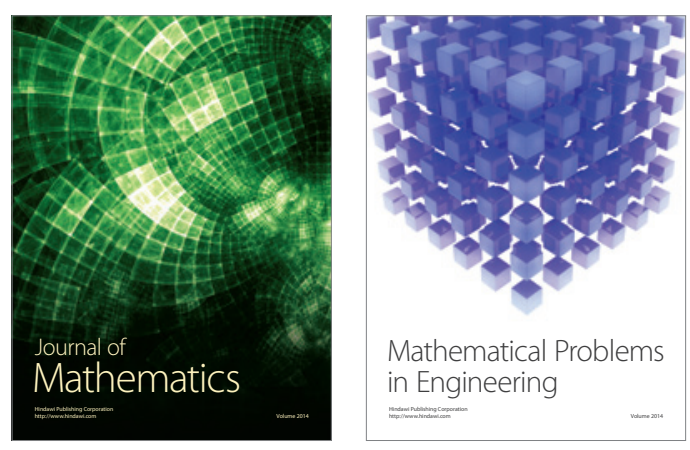

Mathematical Problems in Engineering
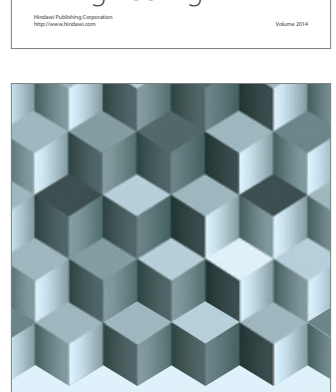

Journal of

Function Spaces
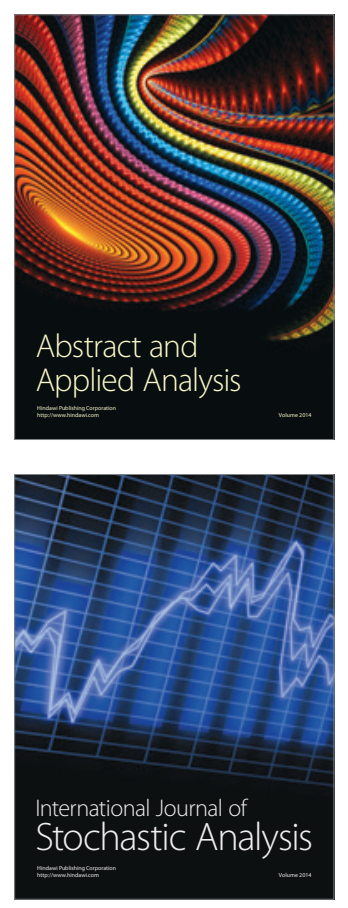

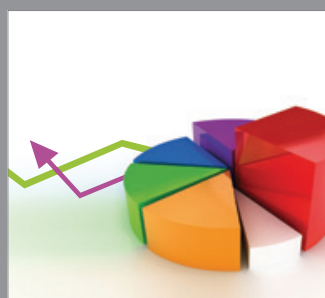

ournal of

Probability and Statistics

Promensencen
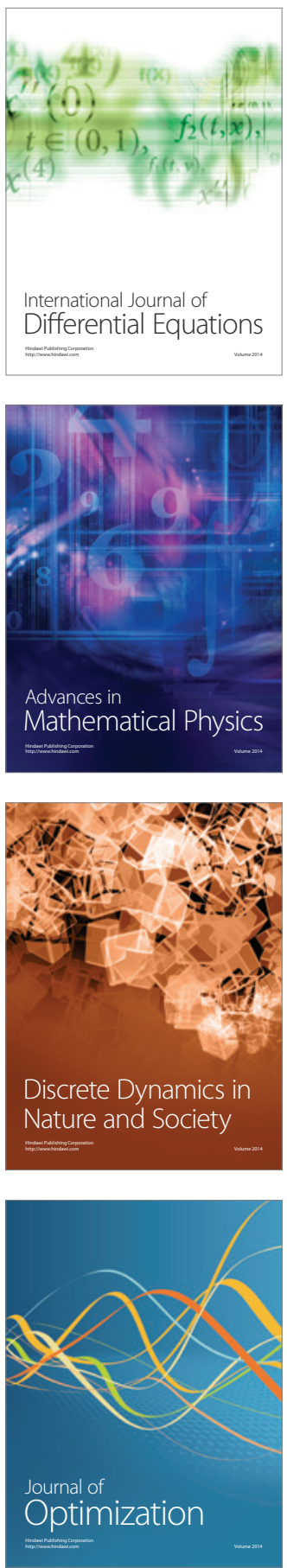